\title{
Realization of a three-functional-layer negative-index photonic metamaterial
}

\author{
G. Dolling and M. Wegener \\ Institut für Angewandte Physik and DFG-Center for Functional Nanostructures (CFN), Universität Karlsruhe (TH), \\ D-76131 Karlsruhe, Germany \\ S. Linden \\ Institut für Nanotechnologie, Forschungszentrum Karlsruhe in der Helmholtz-Gemeinschaft, \\ D-76021 Karlsruhe, Germany
}

Received November 8, 2006; revised December 5, 2006; accepted December 5, 2006; posted December 8, 2006 (Doc. ID 76875); published February 2, 2007

\begin{abstract}
We modify a recently theoretically suggested multilayer negative-index photonic metamaterial design and fabricate corresponding structures with up to three functional layers (seven actual layers) for the first time to our knowledge. Measured transmittance and reflectance spectra agree well with theory. () 2007 Optical Society of America
\end{abstract}

OCIS codes: $160.4760,260.5740$.

Metamaterials are man-made composite materials constructed from subwavelength building blocks that are densely packed into an effective material. Throughout the past few years, photonic metamaterials exhibiting artificial magnetism ${ }^{1-3}$ and a negative refractive index ${ }^{4-8} n$ have become available at optical frequencies. All of these studies, however, investigated single functional layers only (five functional magnetic layers at $6 \mathrm{THz}$ frequency are discussed in Ref. 9). It is well known, e.g., from electronic crystals, that the crystal surface or a monolayer can exhibit properties that are rather different from the bulk. Thus it is relevant to investigate whether the optical constants of a single layer of a negative-index metamaterial really correspond to those of many layers (bulk).

In this Letter we report the successful fabrication of one-, two-, and three-functional-layer negativeindex photonic metamaterials for the first time to our knowledge. Our optical experiments agree well with numerical calculations. This comparison shows that the key features do not change with the number of functional layers.

A single functional layer is shown in Fig. 1(a). As discussed in detail previously, ${ }^{6-8,10}$ the incident light field can excite an antisymmetric current oscillation in the two metal layers of the broad stripes, leading to part of a ring current and hence to a local magnetic dipole moment and to negative magnetic permeability above the magnetic resonance frequency. The electron oscillations along the thin wires lead to a diluted Drude-metal response with negative electric permittivity below the effective plasma frequency. The combination of these two aspects results in a negative index of refraction. The obvious first approach to extend this structure into the third dimension is to stack this building-block metal-dielectric-metal (mdm) with an additional spacer layer between (i.e., mdm-spacer-mdm-spacer-) several or many times. ${ }^{11}$ A simplified design results from altogether eliminating the additional spacer between the building blocks and then merging the adjacent pairs of metal layers into one, leading to the simple layer sequence mdmdmdmdm.... Note that the lattice constant along the stacking direction, $a_{z}$, becomes much smaller than the wavelength of light. This means that the effective-medium approximation is very well justified. Indeed, the design used in the experiments below uses $31 \mathrm{~nm}$ silver and $21 \mathrm{~nm} \mathrm{MgF}_{2}$; hence $a_{z}$ $=52 \mathrm{~nm}$, which is 27 times smaller than the operational wavelength of $1410 \mathrm{~nm}$. Numerical calculations show that the magnetic-dipole moments of the three different functional layers add up to a finite macroscopic magnetic field. The corresponding $\boldsymbol{H}$ field is illustrated in Fig. 1(c). It results mainly from the displacement current, i.e., from $-i \omega \boldsymbol{D}=-i \omega \epsilon_{0} \in \boldsymbol{E}$ in the frequency domain. To illustrate this aspect, Fig. 1(d) shows a snapshot of the $\boldsymbol{E}$ field phase shifted by $90^{\circ}$ with respect to Fig. 1(c).

The silver-based samples are fabricated by standard electron-beam lithography and a lift-off procedure. For details, see our Refs. 3 and 6-8. This fabrication procedure results in nonrectangular side walls, typically with an angle $\alpha$ of $8^{\circ}$ with respect to the substrate normal on all sides. Obviously, this effect becomes particularly influential for thick samples like the multilayer structures of interest here. The electron micrographs shown in Fig. 2 reveal good sample quality.

Our calculations are based on a commercial finitedifference time-domain program package (CST Micro Wave Studio). All geometrical parameters are indicated in Fig. 1; optical material parameters taken are $\mathrm{MgF}_{2}$ refractive index $n_{\mathrm{MgF}_{2}}=1.38$, glass substrate refractive index $n_{\text {substrate }}=\sqrt{2.2}$, and the Drude model for silver with plasma frequency $\omega_{\mathrm{pl}}=1.37 \times 10^{16} \mathrm{~s}^{-1}$ and damping (or collision) frequency $\omega_{\mathrm{col}}=8.5$ $\times 10^{13} \mathrm{~s}^{-1}$. This metal damping strongly influences whether a negative refractive index results from the 
(a)

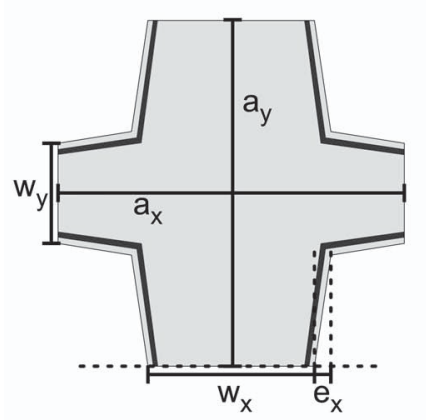

(c)

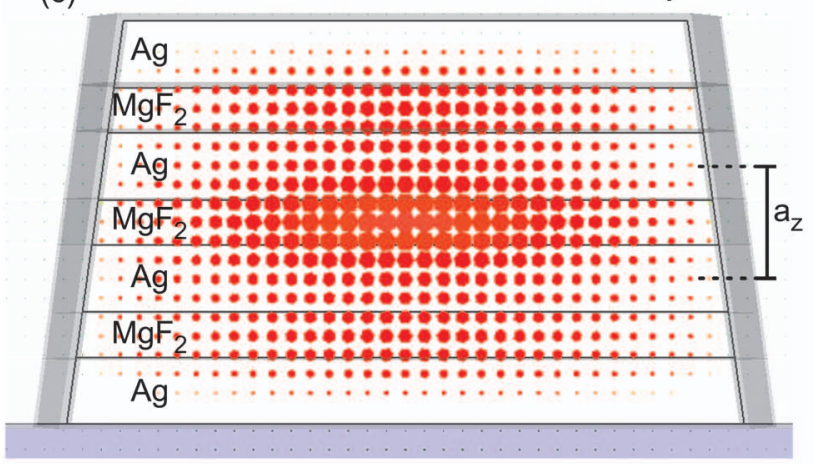

(d)

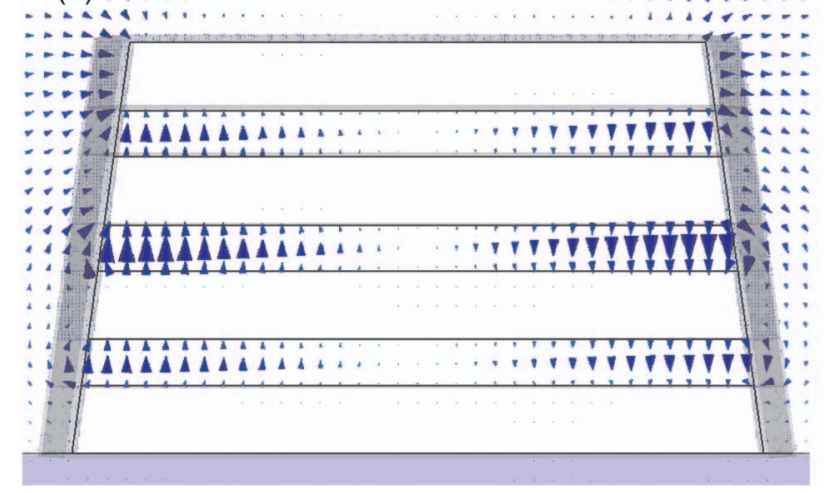

Fig. 1. Scheme of the metamaterial with $N=1$ functional layer under investigation. (a) Top view on one unit cell, (b) side view. The geometrical parameters are indicated and are given by $a_{x}=a_{y}=645 \mathrm{~nm}, w_{x}=318 \mathrm{~nm}, w_{y}=173 \mathrm{~nm}, \alpha$ $=8^{\circ}, t=31 \mathrm{~nm}, s=21 \mathrm{~nm}$, and $e_{x}=e_{y}=14 \mathrm{~nm}$. (c) Snapshot of the $\boldsymbol{H}$ field (linear scale) for $N=3$ and a wavelength of $1430 \mathrm{~nm}$ (compare Figs. 2 and 3) for the plane indicated by the dashed horizontal line in (a). (d) $\boldsymbol{E}$ field (linear scale) for the same plane and wavelength.

parameter retrieval to be discussed below. The chosen damping value is identical to that recently obtained from the comparison with detailed interferometric experiments. ${ }^{7}$ Thus this value can be considered very trustworthy, and we shall not repeat interferometric experiments here. We have previously published various interferometric experiments three times. ${ }^{6-8}$

Figure 2 compares measured and calculated normal-incidence transmittance and reflectance spectra for the two orthogonal linear incident polarizations indicated at the top and for $N=1, N=2$, and $N=3$ functional layers. Three functional layers correspond to seven actual layers, i.e., to the sequence mdmdmdm on top of the glass substrate. Horizontal polar- ization will lead to a negative refractive index (see below); vertical polarization merely serves as a consistency check. The overall agreement between experiment and theory in Fig. 2 is very good-even for the rather complex three-functional-layer sample. Notably, the transmittance for this sample around a wavelength of $1410 \mathrm{~nm}$, where a negative real part of the refractive index occurs (see below), is as large as $60 \%$. Furthermore, the present result for the onefunctional-layer sample are consistent with our previous work. Note that we have used a strictly identical parameter set for the three cases $N=1,2,3$ in our calculations, i.e., identical $w_{x}, w_{y}, a_{x}, a_{y}, \alpha, s, t, e_{x}, e_{y}$, as well as identical optical parameters and identical Drude parameters. Thus, the following retrieval, based on the same theory and parameters, can be considered very trustworthy.

We retrieve the effective optical parameters as usual. ${ }^{12}$ These parameters refer to a fictitious homogeneous film with a thickness corresponding to the total thickness $d$ of the metamaterial stack ( $d$ $=83,135,187 \mathrm{~nm}$ for $N=1,2,3$, respectively) on the glass substrate and with complex field-transmittance and field-reflectance spectra strictly identical to those of the metamaterial. Figure 3 shows that the shape of the retrieved real part of the complex refractive index hardly changes with the number of functional layers $N$ for $N=1,2,3$. The negative real part of $n$ for $N=2,3$ results from the combination of a negative real part of the electric permittivity $\epsilon$ (not

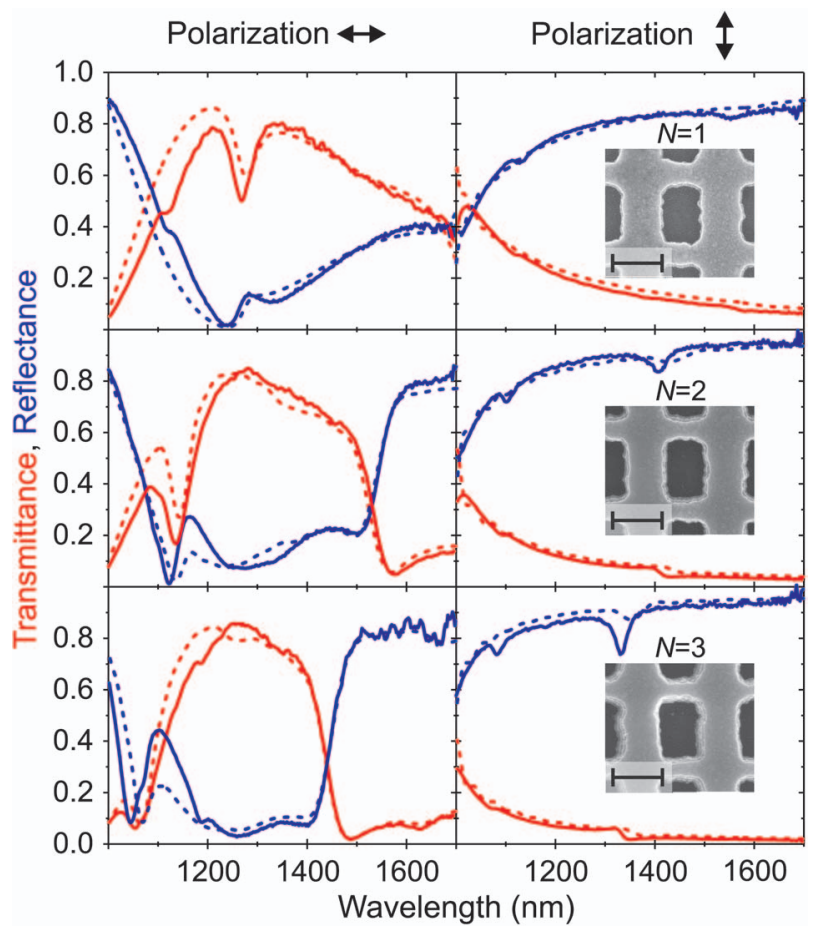

Fig. 2. Measured (solid) and calculated (dashed) normalincidence transmittance (red) and reflectance (blue) spectra for the relevant horizontal incident polarization in the lefthand column. For completeness and as a consistency check, the vertical polarization is shown in the right-hand column. The first, second, and third rows correspond to $N$ $=1,2,3$ functional layers, respectively. Insets, corresponding electron micrographs with $400 \mathrm{~nm}$ scale bar. 


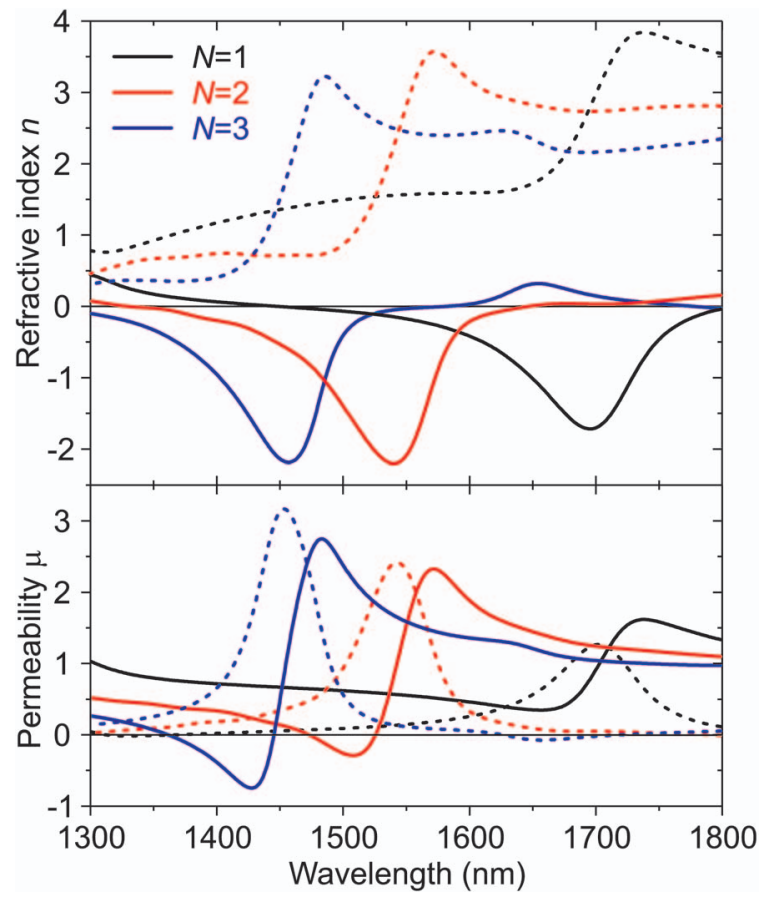

Fig. 3. Effective refractive index $n$ (top) and magnetic permeability $\mu$ (bottom) retrieved from the calculated complex transmittance and reflectance spectra for the same parameters as in Fig. 2; horizontal incident polarization. Real parts, solid, imaginary parts, dashed. The number of functional layers $N$ is indicated.

shown) and a negative real part of the magnetic permeability $\mu$. The increase of the figure of merit (FOM) given by $\mathrm{FOM}=-\operatorname{Re}(n) / \operatorname{Im}(n)$ with $N$ toward a maximum of $\mathrm{FOM} \approx 2.5$ at a wavelength of $1410 \mathrm{~nm}$ should not be taken too seriously, as the detailed behavior depends on for what value of $N$ the response has been optimized. Here we have optimized it for $N=3$. The blueshift of the resonance with increasing $N$ simply results from the trapezoidal shape, i.e., from the finite angle $\alpha$ [see Fig. 1(b)], which directly influences the magnetic resonance frequency. One might have expected three magnetic resonances at three different spectral positions for $N=3$ or a huge inhomogeneous broadening-if the three functional layers were independent. The numerical calculations in Fig. 3 clearly show that this behavior does not occur. The magnetic dipole moment of one functional layer stems from the antisymmetric current oscillation in the two metal layers surrounding one dielectric spacer. Thus one might have naively expected that the magnetic-dipole moments of adjacent functional layers would cancel to some extent. The numerical calculations in Figs. 1(c) and 3 show that this behavior does not occur either. We conclude that interaction effects among the different functional layers are of crucial importance. This conclusion is not surprising in view of the subwavelength distance of the functional layers that introduces a strong coupling via the light field. Furthermore, adjacent functional layers share one common metal layer. These two aspects lead to a cooperative behavior that is very favorable for performance. In particular, it makes the structure robust against varying indi- vidual resonance frequencies, i.e., robust against fabrication tolerances.

In conclusion, we have realized a negative-index photonic metamaterial with three functional layers (rather than just one functional layer). These results are very encouraging in that the measured performance is still close to theory and in that the retrieved optical parameters do not change too much with the number of functional layers - as expected for an ideal metamaterial. Also, for the design presented here, the effective-medium limit is well fulfilled, as the relevant wavelength of light is 27 times larger than the lattice constant in the propagation direction. It is currently not quite clear, however, whether the mentioned cooperative effects will prevail for structures that are many wavelengths in thickness. While our results are a first step toward three-dimensional photonic metamaterials, it should be clear that it is still a long way to truly three-dimensional isotropic negative-index metamaterials at optical frequencies.

We thank Costas M. Soukoulis for discussions and for exciting us about the field of metamaterials. We acknowledge support by the Deutsche Forschungsgemeinschaft (DFG) and the State of BadenWürttemberg through the DFG-Center for Functional Nanostructures (CFN) within subproject A1.5. The research of $\mathrm{S}$. Linden is further supported through a Helmholtz-Hochschul-Nachwuchsgruppe (VH-NG-232). The work is performed within the Karlsruhe School of Optics \& Photonics (KSOP). G. Dolling's e-mail address is gunnar.dolling@ physik.uni-karlsruhe.de.

\section{References}

1. T. J. Yen, W. J. Padilla, N. Fang, D. C Vier, D. R. Smith, J. B. Pendry, D. N. Basov, and X. Zhang, Science 303, 1494 (2004).

2. S. Linden, C. Enkrich, M. Wegener, J. Zhou, T. Koschny, and C. M. Soukoulis, Science 306, 1351 (2004).

3. G. Dolling, C. Enkrich, M. Wegener, J. F. Zhou, C. M. Soukoulis, and S. Linden, Opt. Lett. 30, 3198 (2005).

4. S. Zhang, W. Fan, N. C. Panoiu, K. J. Malloy, R. M. Osgood, and S. R. J. Brueck, Phys. Rev. Lett. 95, 137404 (2005).

5. V. M. Shalaev, W. Cai, U. K. Chettiar, H. Yuan, A. K. Sarychev, V. P. Drachev, and A. V. Kildishev, Opt. Lett. 30, 3356 (2005).

6. G. Dolling, C. Enkrich, M. Wegener, C. M. Soukoulis, and S. Linden, Science 312, 892 (2006).

7. G. Dolling, C. Enkrich, M. Wegener, C. M. Soukoulis, and S. Linden, Opt. Lett. 31, 1800 (2006).

8. G. Dolling, M. Wegener, C. M. Soukoulis, and S. Linden, Opt. Lett. 32, 53 (2007).

9. N. Katsarakis, G. Konstantinidis, A. Kostopoulos, R. S. Penciu, T. F. Gundogdu, M. Kafesaki, E. N. Economou, T. Koschny, and C. M. Soukoulis, Opt. Lett. 30, 1348 (2005).

10. S. Zhang, W. Fan, K. J. Malloy, S. R. J. Brueck, N. C. Panoiu, and R. M. Osgood, Opt. Express 13, 4922 (2005).

11. S. Zhang, W. Fan, N. C. Panoiu, K. J. Malloy, R. M. Osgood, and S. R. J. Brueck, Opt. Express 14, 6778 (2006).

12. D. R. Smith, S. Schultz, P. Marko, and C. M. Soukoulis, Phys. Rev. B 65, 195104 (2002). 